\title{
New insights into the development and differentiation of the human anorectal epithelia. Are there clinical consequences?
}

\author{
Helga Fritsch • Sarah Zehm • Romana Illig • \\ Patrizia Moser $\cdot$ Felix Aigner
}

Accepted: 4 June 2010 / Published online: 20 June 2010

(C) The Author(s) 2010. This article is published with open access at Springerlink.com

\begin{abstract}
Purpose The epithelial lining of the anorectum still raises discussions concerning the levels of transition between the various zones and leads to an incomplete understanding of the immmunoprofile of rectal carcinoma. Since the expression of cytokeratins depends on the epithelial cell-type and the parahox-gene CDX2 is important for the development of the colorectal epithelium, we investigated different cytokeratins and CDX2 in the anorectum of human prenatal stages and in adult normal and neoplastic anorecta.

Materials and Methods The differentiation and spatiotemporal distribution of the epithelial zones were examined in 33 human embryos and fetuses, in a 2-year-old child and four adults. In comparison, 17 specimens of ultralow rectal adenocarcinoma and 4 specimens of anal carcinoma were investigated. Monoclonal antibodies were directed against cytokeratin (CK) 18, 20, 7 and 14 and CDX2.
\end{abstract}

\section{H. Fritsch $(\bowtie) \cdot R$. Illig}

Division of Clinical and Functional Anatomy,

Department of Anatomy, Histology and Embryology,

Innsbruck Medical University,

Muellerstr. 59,

A 6020 Innsbruck, Austria

e-mail: helga.fritsch@i-med.ac.at

\section{S. Zehm}

Clinic of Vascular Surgery, Innsbruck Medical University, Innsbruck, Austria

\section{P. Moser}

Institute of Pathology, Innsbruck Medical University,

Innsbruck, Austria

\section{F. Aigner}

Department of Visceral, Transplant and Thoracic Surgery, Innsbruck Medical University,

Innsbruck, Austria
Results Due to the cytokeratin profile and to CDX2 expression, the different anorectal zones could already be differentiated in human prenatal life. We showed that anorectal epithelial differentiation including the squamous epithelia ran in a craniocaudal direction, and that the anorectal zone was a transitional zone between rectal zone and anal transitional zone where CK 7, 18, 20 and CDX2 are simultaneously expressed. All cases of rectal adenocarcinoma showed positivity for CK 18, 20 and CDX2, and three also labelled for CK 7, whereas CK 14 was only expressed in the cases of anal carcinoma.

Conclusions Our results elucidate the connection between the prenatal pattern and the origin of the different types of anorectal carcinoma.

Keywords Human rectum - Epithelial differentiation . Anorectal tumours $\cdot$ Cytokeratins $\cdot$ CDX2

\section{Introduction}

The epithelial boundaries of the anorectum are of high clinical relevance, as the exact location of pathological processes, especially carcinomas, in relation to these boundaries is crucial for prognosis and therapy [1-3]. The different epithelial zones of the human anorectum have caused numerous discussions concerning their embryonic origin, levels of transition and nomenclature [4]. Whereas it seems to be clear that the upper part of the anorectum belongs to the large intestine and is derived from the entoderm, the lower part is supposed to originate from the ectoderm. In 1914, Johnson [5] postulated that an exact histological determination of the border between entoderm and ectoderm is impossible. Only 10 years ago, O'Rahilly et Müller [6] pointed out that, as a result of developmental 
dislocations, the position of the dentate line does not necessarily allow conclusions concerning developmental processes in the anorectum. Nevertheless, the border between ectodermal- and entodermal-derived anorectal epithelia is still assumed to lie in the area of the dentate line [7-9]. Recently we have revealed the fact that differentiation of the anorectal layers starts after disappearance of the cloacal membrane at the beginning of the seventh week post conceptionem (p.c.). Indications for an interaction between epithelium and mesenchymal structures were shown, whereas an ingrowth of surface ectoderm was not observed. The epithelial differentiation was described as a local process, probably induced by a cross-talk between epithelium and mesenchyme [10].

Up to now, specific markers for the very early embryonic ectodermal and entodermal epithelia are missing. The solution of this question may be a subject of cell biological and experimental studies. From the clinical point of view, however, it is of much more relevance to know which kind of tumour may derive from the different heights of the anorectum. Thus, to get new insights into the developmental differentiation of the anorectal epithelia and probable similarities between the embryonic situation and tumour development in the adult, we used different cytokeratins (CKs) and CDX2 as markers for immunohistochemistry. The expression of cytokeratins is cell-type specific. During malignant transformation their specificity remains conserved in large parts [11-13]. Furthermore, the expression of cytokeratins is specific for different types of epithelial differentiation [14-16]. CK 7 and CK 18/20 are commonly described as markers for simple and columnar epithelium and thus believed to belong to entodermal-derived tissues, whereas CK 14 is a marker for squamous epithelium and thus considered to belong to ectodermal-derived tissue [17]. They have already been applied in several investigations concerning intestinal epithelia of the adult. In our study these cytokeratins were first examined in regard to their ability to demarcate different epithelial sections within the developing anorectum. The results were compared with the normal situation in the adult and with cases of both low rectal and anal carcinoma.

CDX2 belongs to the family of parahox genes and encodes an intestine-specific transcription factor which is important for the regulation of proliferation and differentiation of intestinal epithelial cells [18]. It is expressed in the nuclei of entodermal-derived epithelial cells from duodenum to rectum. Previous studies showed CDX2 expression in normal colonic mucosa and in most colorectal adenocarcinomas and metastases, but it is rarely seen in tumours of other sites [19-21]. In the present study, we examined CDX2 expression in the human anorectal epithelia during development, in the adult and in carcinomas of both the low rectal and anal region.
It was the purpose of this study to create an actual basis for cellular origin and differentiation of the anorectal region including carcinomas. The hypothesis of the underlying study is that the prenatal situation indicates the origin of the different types of carcinomas in the adult anorectum.

\section{Materials and methods}

Differentiation and spatiotemporal distribution of the different epithelial sections of the anorectum were examined by means of conventional histology and immunohistochemistry using monoclonal antibodies directed against cytokeratins (CK) 18 , 20, 7 and 14 as well as CDX2.

Thirty-three human embryos and fetuses (8th-33rd week p.c.), sections of a 2-year-old child and of four adults (36 years old male, 65 years old male, 76 years old male and 74 years old female) were studied microscopically.

The embryonic and fetal specimens were provided by the collection of the Department of Anatomy, Histology and Embryology, Innsbruck, or obtained from legal abortions collected from different gynecologists having received parental consent. The adult specimens were also taken from the Department's collection and had no intestinal disorders or malformations.

Specimens were fixed in 7\% formaldehyde in PBS $(0.2 \mathrm{M})$. After paraffin embedding in a routine histological infiltration processor (Miles Scientific Inc., Naperville, IL, USA), specimens were cut in $4-\mu \mathrm{m}$ sections in sagittal or axial planes by means of a Microm ERGO Star Rotationsmicrotome (Microm, Walldorf, Germany). The sections were dried overnight, dewaxed with xylene, rehydrated in graded alcohol series, and every 10th to 20th section of a series was stained with haematoxylin and eosin (HE).

For immunohistochemical study the Discovery XT automated staining system by Ventana Medical Systems Inc. (Ventana, Strasbourg, France) was used. After dewaxing and rehydrating, the antigen retrieval was started by heat (for CK 18, 20 and 14 staining) or protease (for CK 7 staining) induced epitope unmasking while the sections were immersed in citrate buffer. The slides were incubated with prediluted monoclonal antibodies recognizing CK 7 , 14, 18, 20 (Linaris, Germany) for 60 minutes and biotinylated secondary antibody (universal, IGG antimouse, antirabbit) (Ventana, Strasbourg, France) for 30 minutes. Subsequently, the antigen-antibody complex was fixed with $0.05 \%$ glutaraldehyde in $0.9 \%$ sodium chloride. Antibody binding sites were visualised by means of a 3.3-diaminobenzidine (DAB) detection kit (according to the manufacturer's protocols).

For CDX2 staining, we used Histoprime ${ }^{\circledR}$ Cdx2, a monoclonal mouse antibody, ready-to-use clone $\mathrm{Cdx} 2-88$ (Linaris, Wertheim-Bettingen, Germany). Subsequent to 
primary antibody incubation for half an hour and secondary antibody incubation for 15 minutes, DAB detection kit was applied according to the manufacturer's guidelines.

All sections were counterstained with haematoxylin and blueing reagent. Dehydration in ethanol and xylene series was carried out, and finally the sections were mounted permanently by a cytosol embedding medium (Microm, Walldorf, Germany). Negative controls without primary antibody did not show antibody-induced labelling.

In a second part of the study, tumour blocks of 17 patients (mean age 74 years, range 57-86 years) with ultralow rectal cancer (pT2, N0, M0, GII-pT3, N1, M0, GIII) following abdominoperineal resection or anterior resection of the rectum without preoperative radiochemotherapy and resection specimens of four patients (mean age 73 , range $62-82$ years) with anal squamous cell carcinomas with preoperative radiochemotherapy were processed accordingly. The samples were obtained from the Institute of Pathology, Innsbruck.

\section{Results}

The following subdivision is equivalent to the nomenclature and subdivision of the anorectum proposed by Fenger [4]:

The rectal zone (RZ) is characterized by regularly arranged colonic crypts (Fig. 1). The anorectal zone (ARZ) is a continuation of the rectal mucosa with shortening and irregularity of the crypts (Fig. 1). The anal

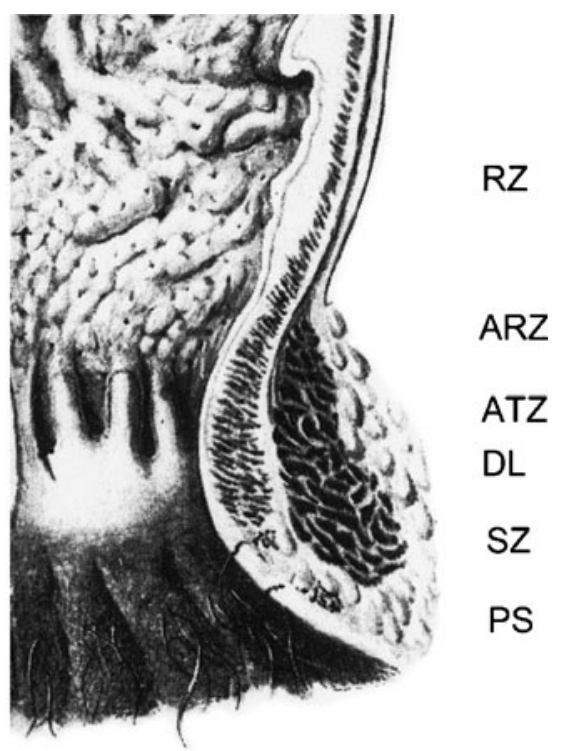

Fig. 1 Schematic drawing of the anorectum and its mucosa transitional zone (ATZ) is characterized by anal columns and sinuses and has epithelial variants (columnar, cuboidal and squamous cells) (Fig. 1), which in most cases end at the dentate line (DL). The length of both, ARZ and ATZ, is variable. The squamous zone (SZ) is covered with uninterrupted squamous epithelium (Fig. 1) and gradually merges into the perianal skin (PS), covered by keratinized squamous epithelium (Fig. 1).

Nine- to eleven-week-old fetuses

\section{Rectal zone}

Whereas the rectum showed a stratified columnar and regularly arranged epithelium without goblet cells in 8-week-old embryos [10], protuberances of the epithelium and the propria, that is, first signs of transitional villi, as well as goblet cells appeared in 9/10-week-old fetuses (Fig. 2a). Positive labelling for $\mathrm{CK} 18$ was observed throughout all rectal epithelial cell layers (Fig. 2b). They were negative for CK 7 (Fig. 2c) in the RZ. Whereas negative reaction was found for CK 14 (data not shown), positive staining reaction was demonstrated for CDX2 (Fig. 2d). Some superficial epithelial cells labelled positive for CK 20 (Fig. 2e).

\section{Anorectal zone}

In the ARZ, positive labelling for CK 18 was detected (Fig. 2b, g), and also some isolated positive cells for $\mathrm{CK}$ 7 with an accumulation near the transitional zone (Fig. 2c).

\section{Anal transitional zone}

The transitional zone was lined by two- to five-layered stratified cuboidal epithelium (Fig. 2f) with vertical surface cells in the cranial area and more flattened caudally. The differentiation of the anal columns and sinuses already started in 9/10-week-old fetuses. The epithelium of the transitional zone showed strong panepithelial reaction for CK 18 (Fig. $2 \mathrm{~g}$ ) as well as for CK 7 (Fig 2h), with pronounced immunoreactivity in the suprabasal layers. In this early stage, CK 14 was not detected in epithelial cells of the transitional zone (data not shown). Negative reaction was found for CK 20 (data not shown) and CDX2 (Fig 2i).

\section{Squamous zone and perianal skin}

The anal canal was lined by uninterrupted stratified squamous epithelium (Fig. 2j). The superficial cells were 

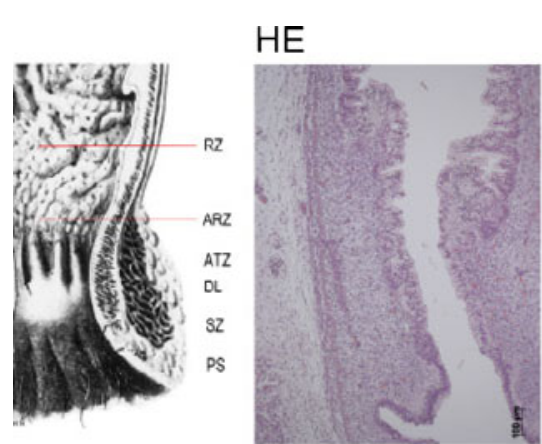

a
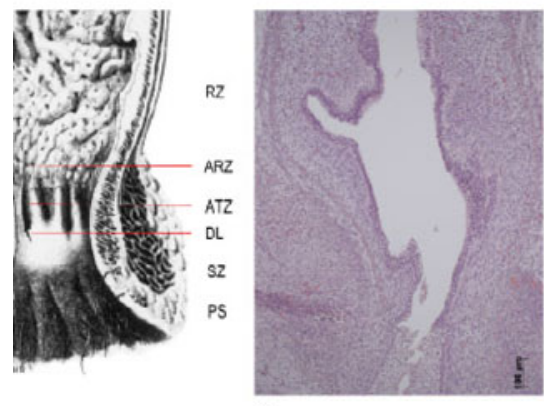

f
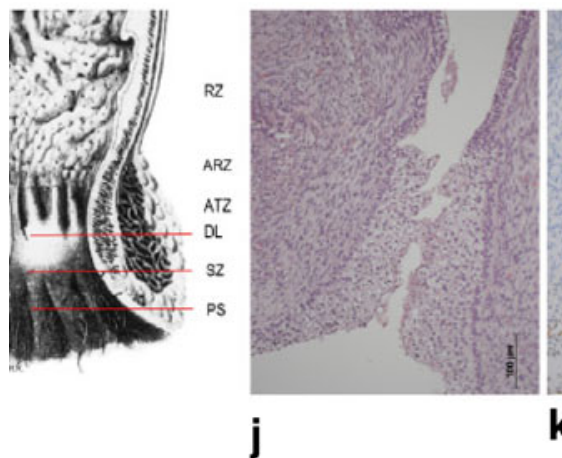

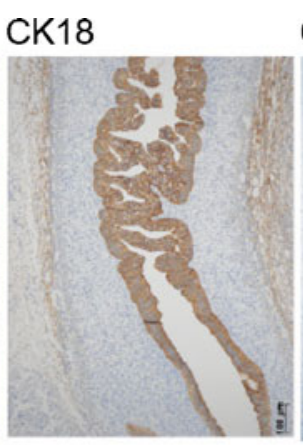

b

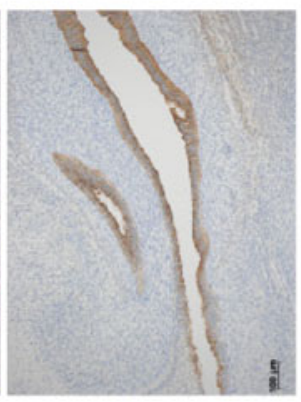

g

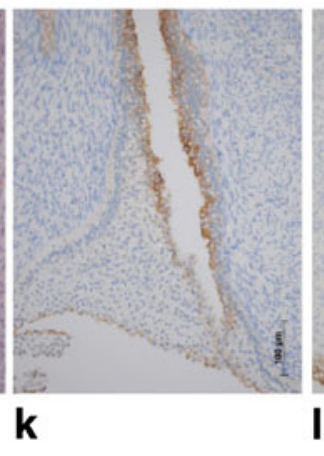

CK7

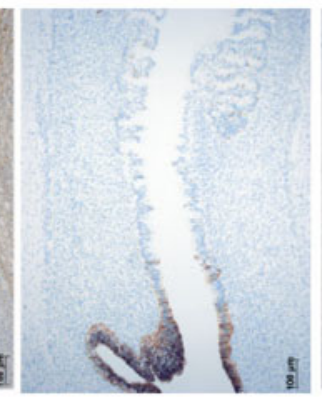

C

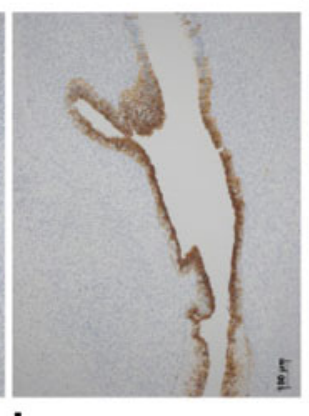

h

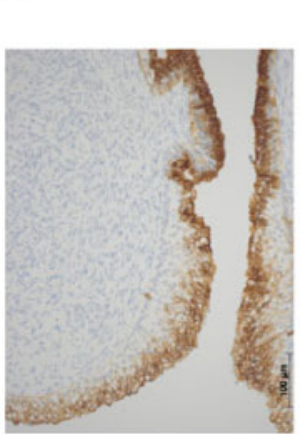

I

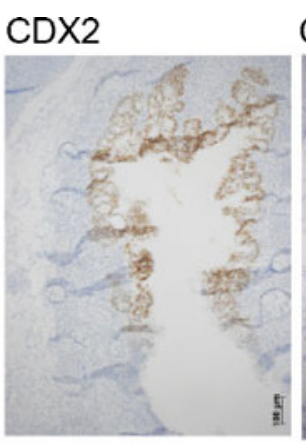

d

CDX2

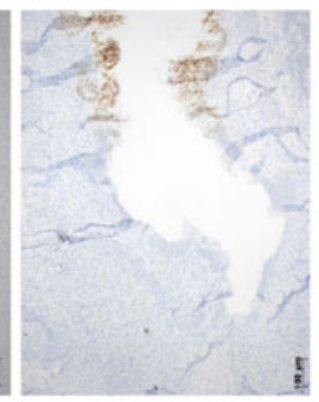

i

\section{CK14}

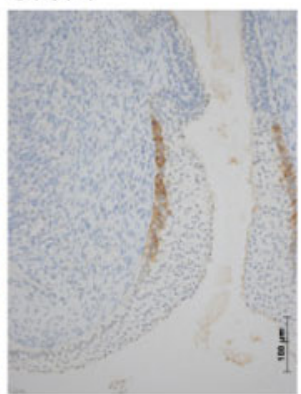

m
CK20

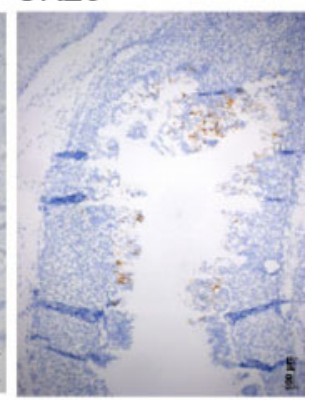

e

Fig. 2 a-m Series of sagittal sections through the anorectum of a 9- to 10-week-old human fetus. a-e RZ, $\mathbf{f}-\mathbf{I}$ ARZ and ATZ, j-m DL, SZ and PS

positive for CK 18 (Fig. 2k) and strongly positive for CK 7 (Fig. 21). Whereas negative reaction was found for CDX2 (not shown), CK 14 affinity was first seen and restricted to basal layers (Fig. $2 \mathrm{~m}$ ) in the future non-keratinizing zone.

Twelve- to fifteen-week-old fetuses

\section{Rectal zone}

In 12-week-old fetuses, the rectal surface was covered by transitional villi, lined by a one- to two-layered columnar epithelium with goblet cells (Fig. 3a). The epithelium showed positive reaction for $\mathrm{CK} 18$ (Fig. 3b) and negative reaction for CK 7 (Fig. 3c) as well as for CK14 (not shown). The labelling for CDX2 was positive, especially in the epithelium covering the bottoms between the villi (Fig. 3d). Labelling for CK 20 (Fig. 3e) was particularly positive in the surface epithelium and was spread in the same area as CDX2.

\section{Anorectal zone}

This area was characterized by vanishing villi (Fig. 3a, f). CK 18 (Fig. 3b, g) and CK 7 (Fig. 3c, h) labelling was positive; some scattered CDX2-positive cells (Fig. 3d, i) and CK 20, respectively, (Fig. 3e, j) were found.

\section{Anal transitional zone}

Whereas the epithelia and their immunoreactivity did not change (Fig. $3 f-j$ ) in comparison to the younger stage, the differentiation of the columnar region into anal columns and sinuses was evident. 

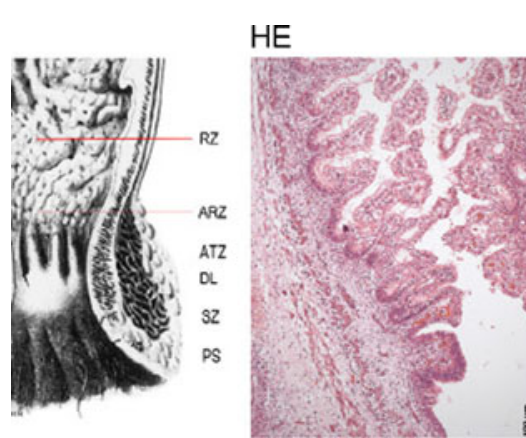

a
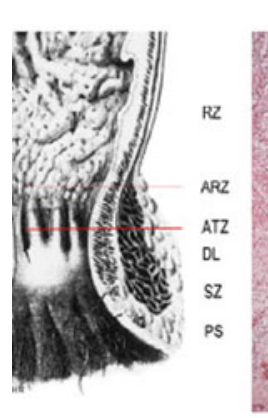

CK18

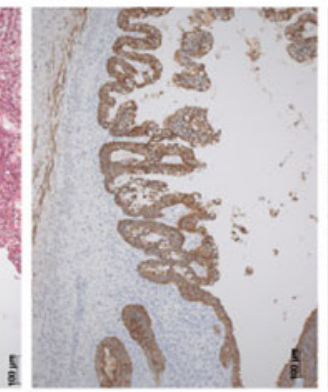

b

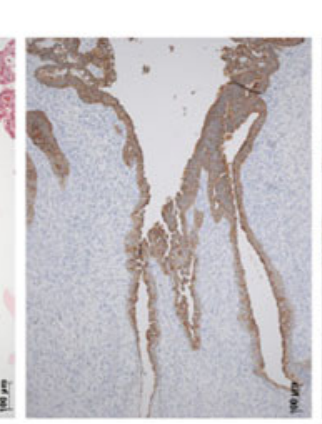

g

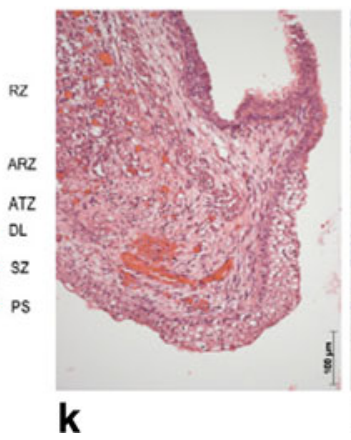

CK7

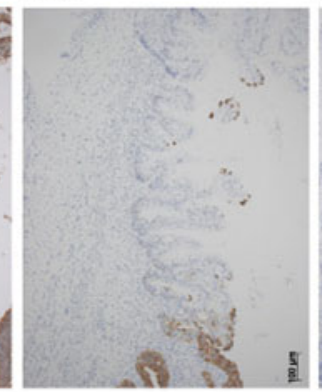

c

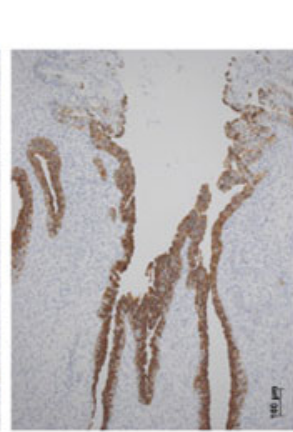

h
$\operatorname{CDX} 2$

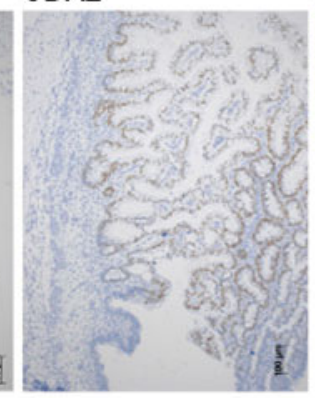

d

$\operatorname{CDX} 2$

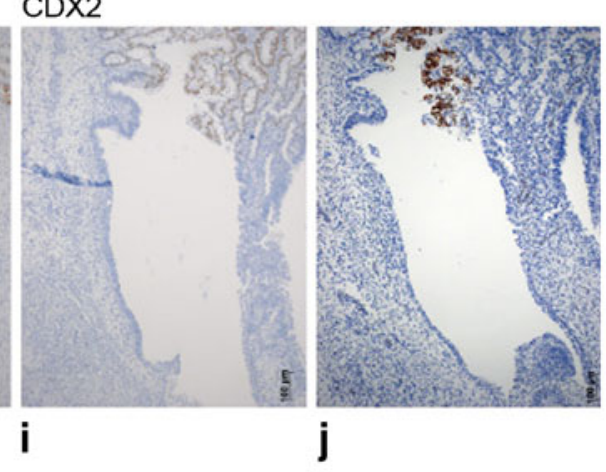

CK14

n

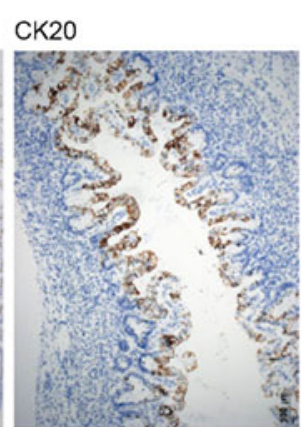

e

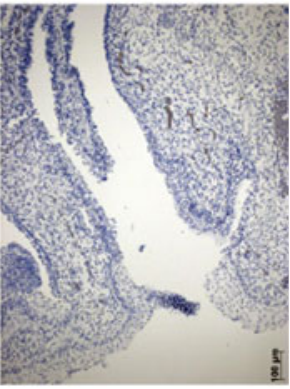

o

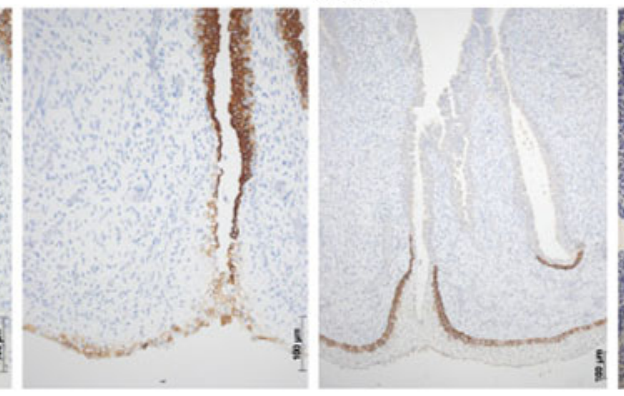

m

Fig. 3 a-o Series of sagittal sections through the anorectum of a 12-week-old human fetus. a-e RZ, $\mathbf{f}-\mathbf{j}$ ARZ and ATZ, k-o DL, SZ and PS

\section{Squamous zone and perianal skin}

The squamous epithelium (Fig. 3k) of the anal region showed only weak reactivity for CK 18 (Fig. 31), superficial cells were still positive for CK 7 (Fig. $3 \mathrm{~m}$ ), and basal cell layers were strongly positive for CK 14 in the anal squamous epithelia (Fig. 3n) and in the sinuses. No labelling was observed for CDX2 (not shown) and for CK 20 (Fig. 3o). In the SZ skin appendages were first seen about the 14th week.

Sixteen- to twenty-four-week-old fetuses

\section{Rectal zone}

The transitional villi were vanishing and regular crypts appeared. The overall single-layered columnar epithelium contained numerous goblet cells (Fig. 4a) and showed positive and consistent labelling for CK 18 (Fig. 4b), whereas positive reaction for CDX2 (Fig. 4c) seemed to be more concentrated at the bottom of the crypts. CK 20-positive reaction was found within the wall of the crypts (Fig. 4d).

\section{Anorectal zone}

As in the earlier stages, the ARZ was characterized by an irregular surface with columnar epithelium and some interspersed goblet cells (Fig. 4e), positive labelling for CK 18 (Fig. 4f), CK 7 (Fig. 4g), CK 20 (Fig. 4h), but only few CDX2 positive cells (not shown).

\section{Anal transitional zone}

The major feature of the transitional zone consisted of a layered cuboidal epithelium (Fig. 4i) and stratified squamous epithe- 
lium at the bottom of the sinuses. Weak positive reaction for CK 18 was restricted to the cranial part of this zone (Fig. 4j), whereas CK 7-labelled cells appeared throughout the whole transitional zone (Fig. 4k). Positive staining for CK 14 was found in the basal cell layers of the squamous epithelium (Fig. 41), especially at the bottom of the sinuses. CK 20 and CDX2 reactions were negative (data not shown).

\section{Squamous zone}

The epithelial border between the transitional zone and anal stratified squamous epithelium stood out clearly and marked the DL (Fig. 41). The anal squamous epithelium labelled weakly or negatively for CK 18 (Fig. 4m) and CK 7 (not shown) and positively in its basal layers for CK 14 (Fig. 4n) which were also $\mathrm{CK} 14$ positive at the bottom of the anal sinuses.

\section{Perianal skin}

The border between non-keratinizing (SZ) and keratinizing epithelium (PS) became evident (Fig. 4l) due to first signs of keratinization in the 16th week. Labelling for CK 18 (Fig. $4 \mathrm{~m}$ ) and CK 7 were mostly negative, but CK 14 labelled positive in the basal layers of all stratified squamous epithelia (Fig. 4n).

Twenty-five-week to thirty-three-week-old fetuses

\section{Rectal zone}

The epithelium of the regular crypts (Fig. 5a) labelled positive for CK 18 (Fig. 5b). CDX2 expression was observed at the bottom of the crypts and partially in the walls (Fig. 5c). Strong labelling for CK 20 was evident within the walls and at the surface, but not at the bottom of the crypts (Fig. 5d).

\section{Anorectal zone}

The labelling within the epithelium of the irregular crypts (Fig. 5e) was similar to that of the RZ concerning CK 18 (Fig. 5f), CDX2 (Fig. 5g) and CK 20 (Fig. 5h), but in contrast remarkably positive for CK 7 (not shown).

\section{Anal transitional zone}

Regular columns and sinuses were seen (Fig. 5i). Intensive CK 7-positive staining was detected (Fig. 5j).

\section{Squamous zone and perianal skin}

The basal cell layers of the non-keratinizing and the keratinizing epithelia (Fig. 5k) were positive for CK 14 (Fig. 51).
Fig. 4 a-k Series of sagittal sections through the anorectum of a 16-week-old human fetus (a-h) and a 20-week-old fetus (i-n). a-d RZ, e-h ARZ and ATZ, i-k ATZ and DL, $\mathbf{l}-\mathbf{n}$ SZ and PS

\section{Postnatal stages/adult}

No structural differences in the anorectal epithelia (Fig. 6a, e, i, k) were found. Cytokeratin 18, 20 and 7 (Fig. 6b, d, f, $\mathrm{h}, \mathrm{j}$ ) as well as CDX2 (Fig. 6c, g) expressions showed no differences compared to the late fetal stages. Panepithelial CK 14 expression was found in the SZ and PS (Fig. 61).

\section{Ultralow rectal tumours}

Seventeen tissue probes from patients with clinical-staged low rectal adenocarcinoma were investigated (Fig. 7a). The tumour tissue was strongly positive for CK 18 (Fig. 7b), negative for CK 14 (not shown) and in most cases (15) negative for CK 7 (Fig. 7c). The two CK 7-positive samples showed sphincter infiltration. All rectal adenocarcinomas displayed positive labelling for CDX2 (Fig. 7d).

\section{Anal tumours}

In correspondence with our findings in healthy adult anal epithelia, the examined anal squamous cell carcinomas (Fig. 7e) of the four investigated probes were negative for CK 18 (Fig. 7f) and CK 7 (Fig. 7g) but remarkably positive for CK 14 (Fig. 7h). They all were negative for CDX2 (not shown).

\section{Discussion}

The present study was undertaken to examine the development and differentiation of the anorectal epithelia and to compare the results with the histomorphological characteristics of anorectal tumours. We report that:

a. Due to the cytokeratin profile and to the expression of CDX2, the different zones of the anorectum can be recognized early in human prenatal life;

b. Epithelial differentiation runs in a craniocaudal direction;

c. The ARZ is an area of epithelial changes; and

d. We explain why CK7, CK20 and CDX2 can simultaneously be expressed in rectal adenocarcinomas.

Following the detailed investigation of Johnson [5], our study is the first to contribute to the histogenesis of the anorectal epithelia. Moreover, we compare the situation in human prenatal stages with adult specimens as well as with ultralow rectal and anal carcinoma. In the last decade, some morphological studies concerning the early development of the human anorectum were published that-in connection 

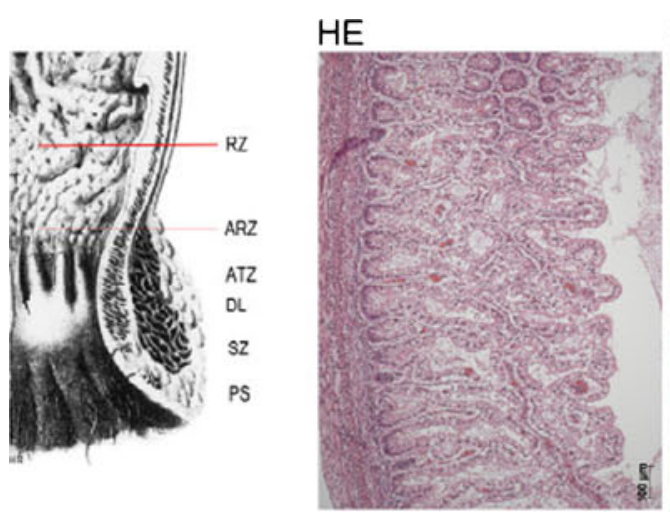
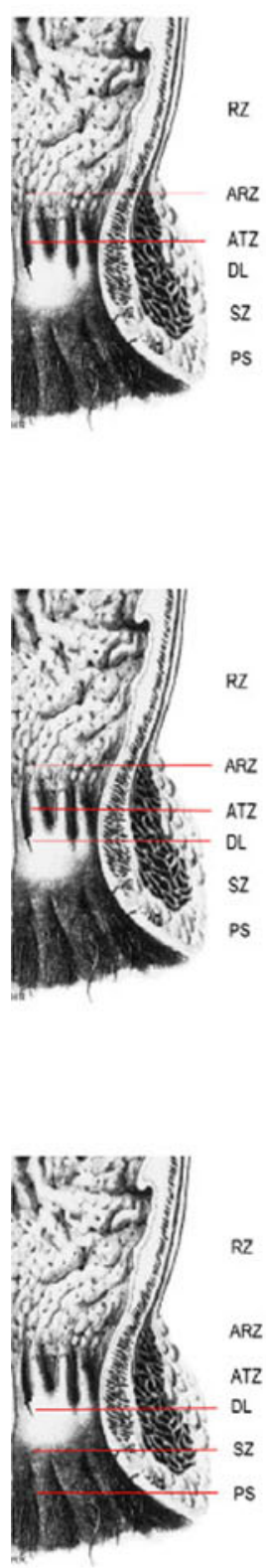

a
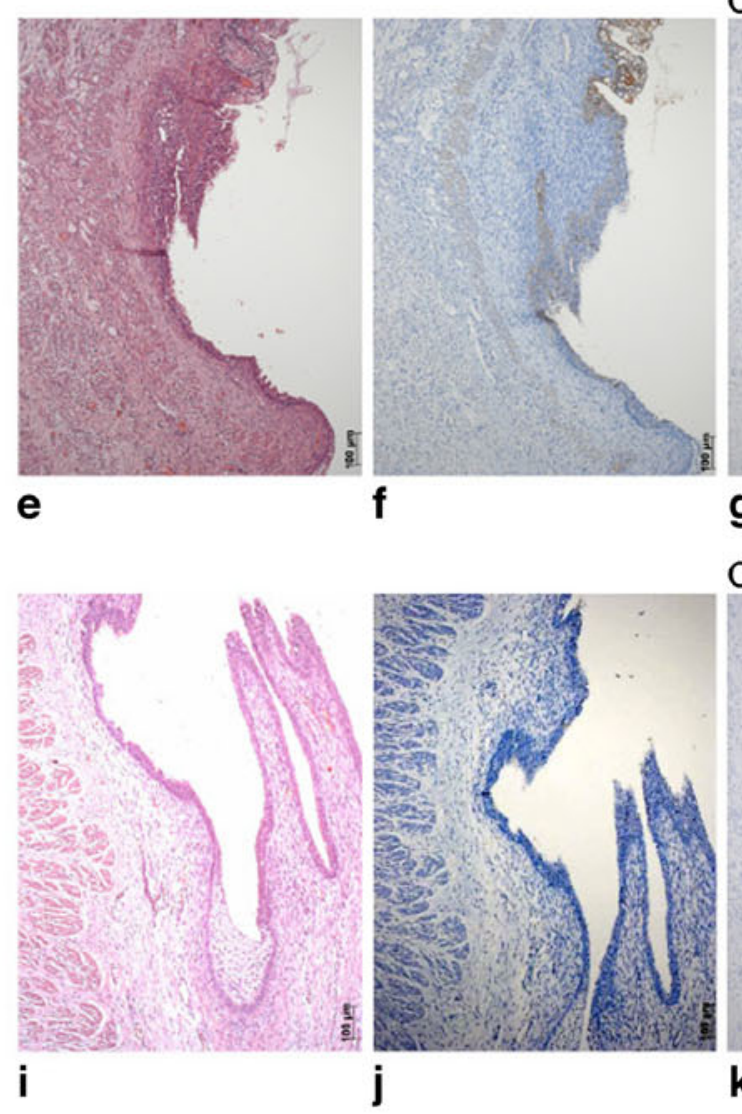

b

g

CK7
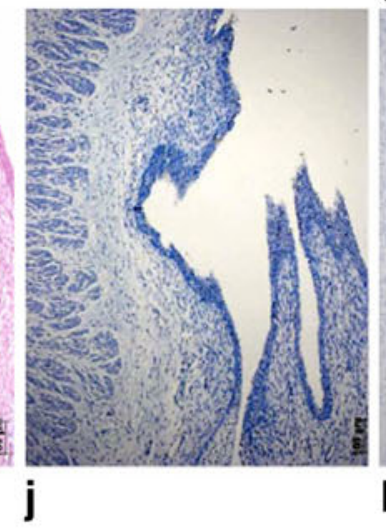

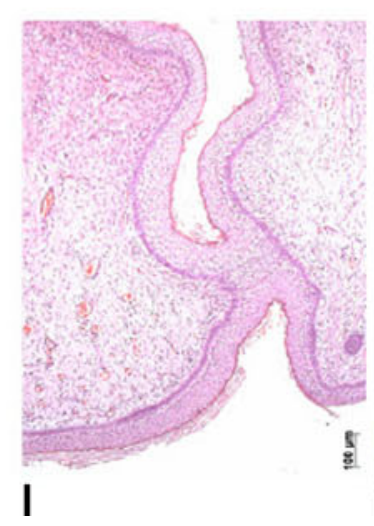

C

CK7

k

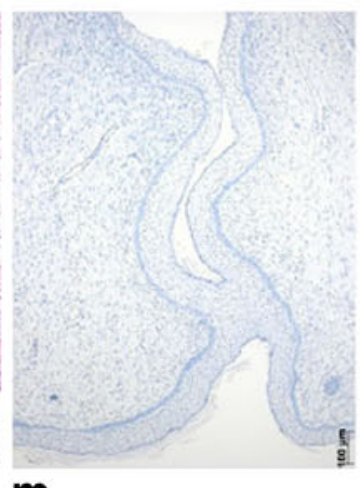

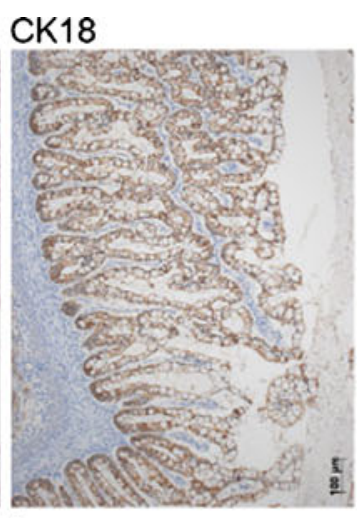

CDX2
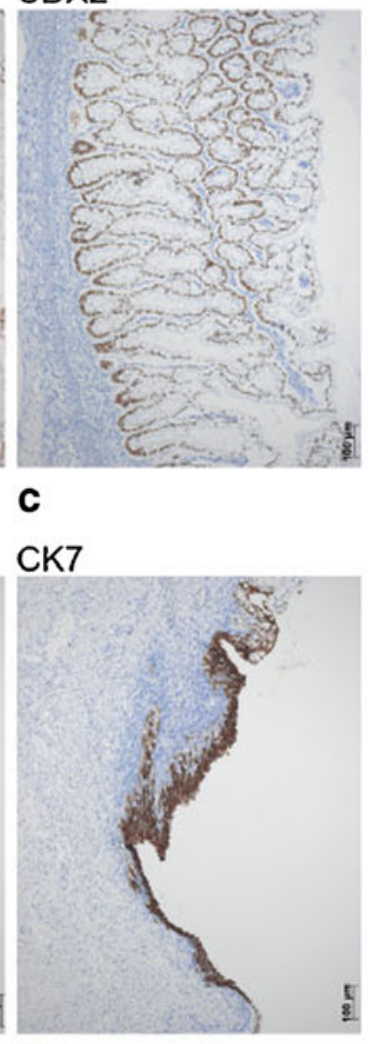

th

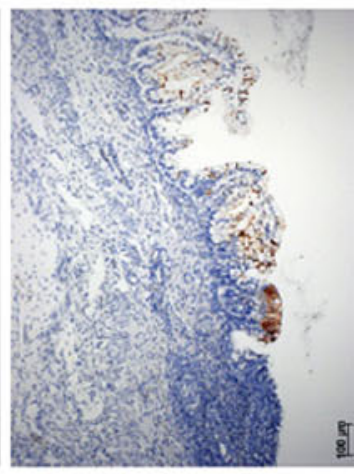

h

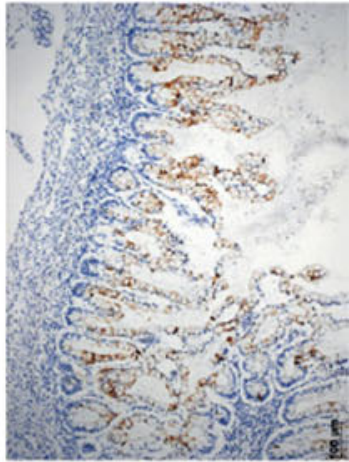

d

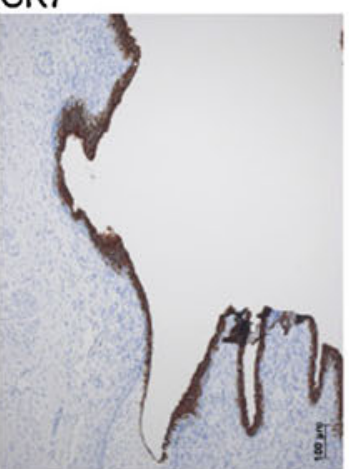

CK14

m

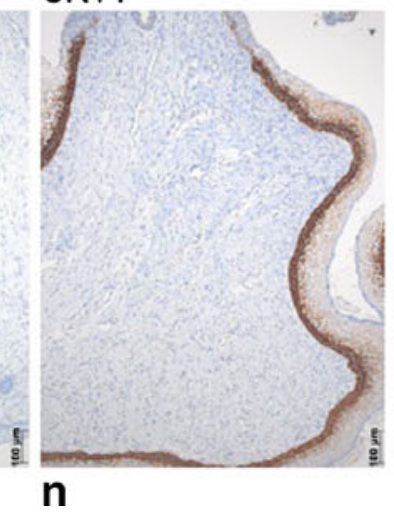



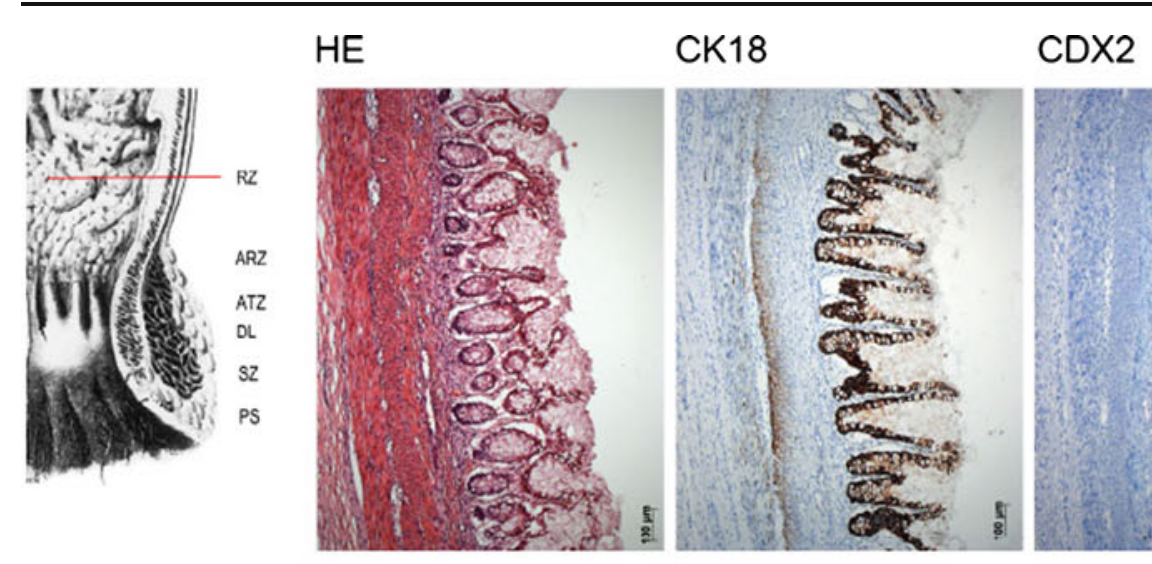

CK20

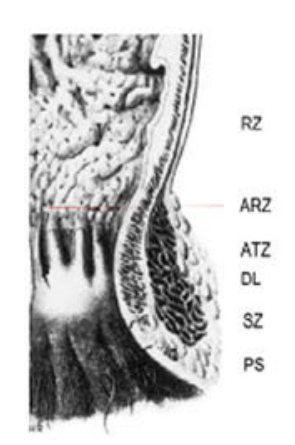

a

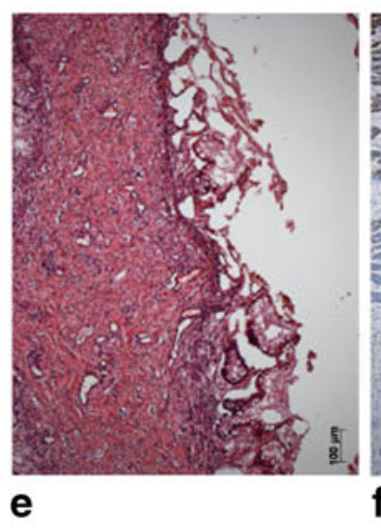

b

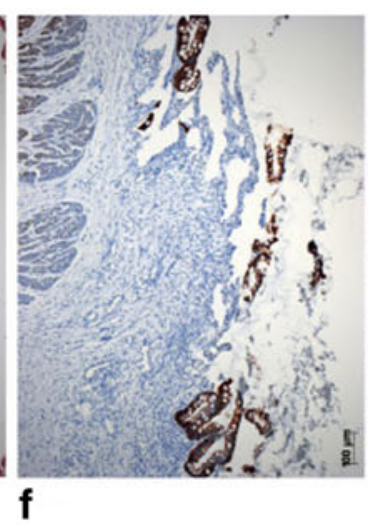

CK7
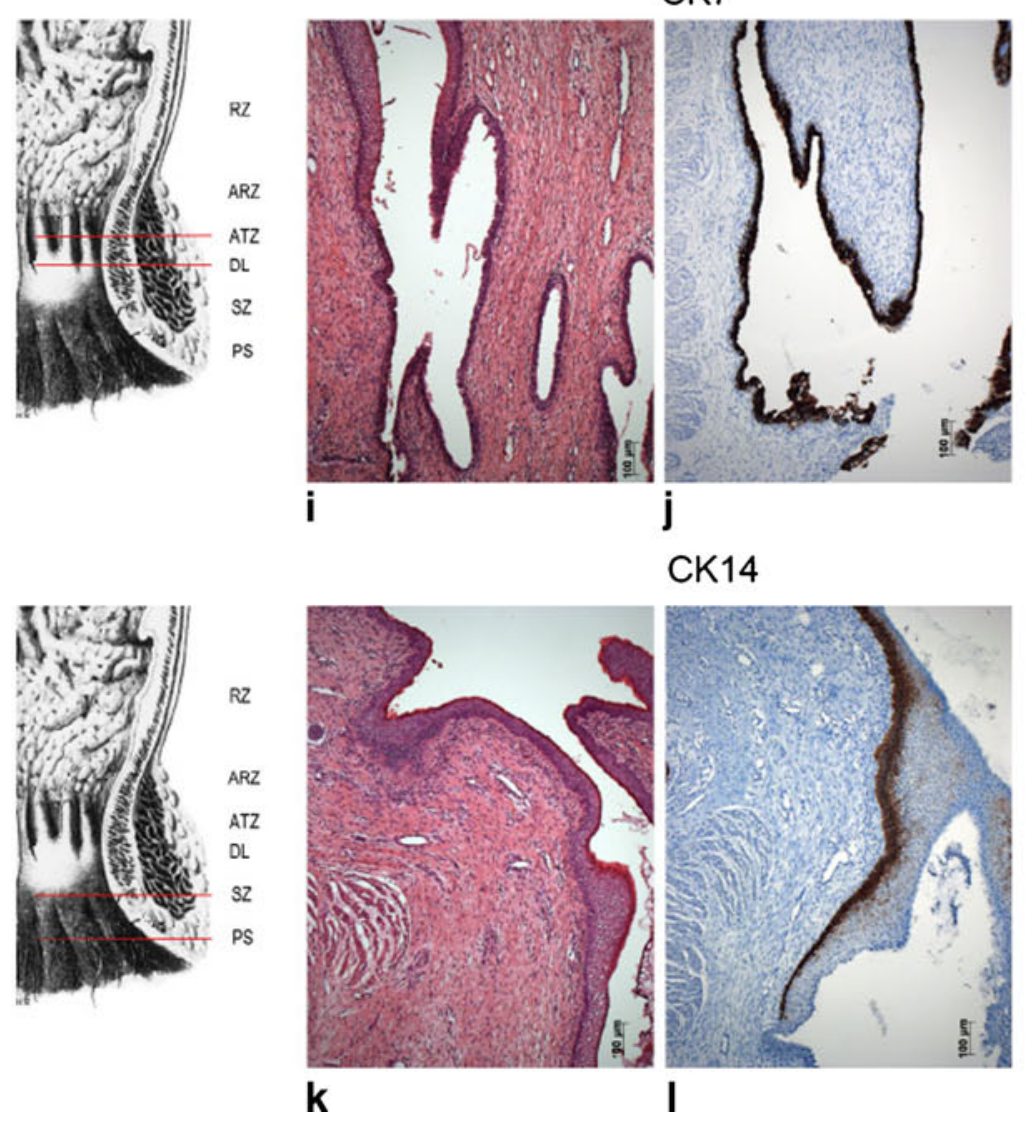
Fig. 5 a-m Series of sagittal sections through the anorectum of a 25-weekold human fetus. a-d RZ, e-g ARZ, $\mathbf{h}-\mathbf{j}$ ATZ and DL, $\mathbf{k}-\mathbf{m}$ SZ and PS

with the fate of the cloaca and the cloacal membranemainly contributed to the debate of the entodermal or ectodermal origin of the different epithelia [22-24]. All these publications have the same weakness: they are only based on normal HE histology. Thus, the results are purely descriptive, mostly speculative and therefore, in regard to their contents, without any connection to new molecular biological findings in laboratory animals or to clinical pathological aspects. To be able to gain new aspects as to the heterogeneity of the different anorectal epithelia, we are the first to include immunohistochemistry of different cytokeratins and of CDX2 expression in all human prenatal stages.

Based on Fenger's [4] clearly arranged subdivision of the anorectum into five epithelial zones, the results of our study can be summarized as follows:

As everywhere in the large intestine, the rectal mucosa has transitional folds in early fetal life [25]. Starting in the eighth week, the epithelium of the RZ is positive for CK 18 and CDX2 during all following stages of human development, and for $\mathrm{CK} 20$ from the tenth week onwards. Rectal CK 7 positivity is obvious only in the ARZ where CK 20 and CDX2 staining vanishes. It is the most important finding that the ARZ already exists in prenatal life. This is why the ARZ must be considered as an area of epithelial changes and not as a metaplastic one. It differs from the RZ as to $\mathrm{CK} 7$ expression.

The epithelium of the ATZ mostly consists of stratified cuboidal epithelium in prenatal stages [24] and is characterized by positive labelling for CK 7 . Anal valves are covered with squamous epithelium and thus show CK 14-positive reaction.

According to our results and to former studies $[5,26]$, the rectal columns differentiate in the late embryonic and early fetal period and arise from the entoderm [10, 22]. The height of the columns is covered with non-keratinizing stratified squamous epithelium only in the postnatal stages as was already shown by Hollinshead [27]. Both anal stratified epithelia are positive for CK18 and CK7 in early stages before gaining CK14 positivity from the ninth week onwards.

Our results clearly show that cell differentiation in the anorectal region does occur craniocaudally, regardless of where the cells take their origin from, and this is also true for the differentiation of the anal squamous epithelium. This backs up the results of our last study where we supposed that "an ingrowth of surface ectoderm is not evident ... and that the epithelial differentiation is a locally organized process and induced by the interaction with the mesenchyme ..." [10]. Contrary to another study [28] whose authors only elaborate on the introversive transfer of the epithelium and on that basis speculate that the epithelium is growing inwards from the outside, we are able to prove beyond doubt that the differentiation starts inside and proceeds externally.

When comparing the results of the human fetuses and adults with the cases of low rectal and anal tumours, we expected to find a cytokeratin profile of the carcinoma cells that is similar to its native epithelium, i.e. we expected CK 18/CK 20- and CDX2-positive labelling in the low rectal carcinomas and $\mathrm{CK} 14$ positivity in tumours deriving from the squamous epithelium of the anal canal below the DL. The rectal tumour probes we included in the present study showed the expected labelling for CK 18/20 and CDX2. In three cases we found an additional positivity for $\mathrm{CK} 7$ which - in contrast to former studies postulating that the presence of CK 7 can be used to exclude a rectal origin of tumours and metastases - is not astonishing as the tumours are characterized as ultralow rectal ones that originate in the ARZ where positive labelling for CK 7 normally occurs. These important findings are in accordance with a recent study [29] which revealed that CK 7 can be expressed in rectal adenocarcinomas, and that the combination of $\mathrm{CK}$ 20/7 with CDX2 is an excellent one securely to identify metastatic rectal carcinomas. This might be helpful in discriminating rare anal malignant conditions like adenocarcinoma of the anal canal from the more common squamous cell carcinoma on the one hand and ultralow adenocarcinoma of the rectum on the other hand. Adenocarcinoma of the anal canal is rare and there is often diagnostic doubt between true anal canal cancer, most likely arising from the anal glands that develop from the rectal epithelium [10] and direct invasion from very low rectal cancers [30]. Changes in the expression of certain CKs provide an indication of the differentiation stage (dedifferentiation) of a tumour which is comparable to prenatal stages of differentiation and the respective expression pattern [31].

Our study successfully aimed to show that the demarcation line between the five epithelial zones is more distinct in some places than in others: whereas it is rather transient between the RZ and the ARZ, on the DL it is clearly delimited in all stages of development. Below the DL the demarcation between the keratinizing stratified squamous epithelium of the anal canal and the non-keratinizing stratified squamous epithelium of the rectum is clearly visible from the 16th week of development. According to our qualitative results, the extent of the different anorectal sections, especially of the ATZ, is variable and differs interindividually [4]. Therefore, in the case of a tumour, it is most important to document the exact location macroscopically as well as microscopically.

CDX2 discontinues to be expressed at the borderline between the RZ and the ATZ, i.e. in the ARZ. Cranial to the ARZ, transitional folds - typical for the intestinal identityappear in early fetal life. Thus, up to this line, the intestine 

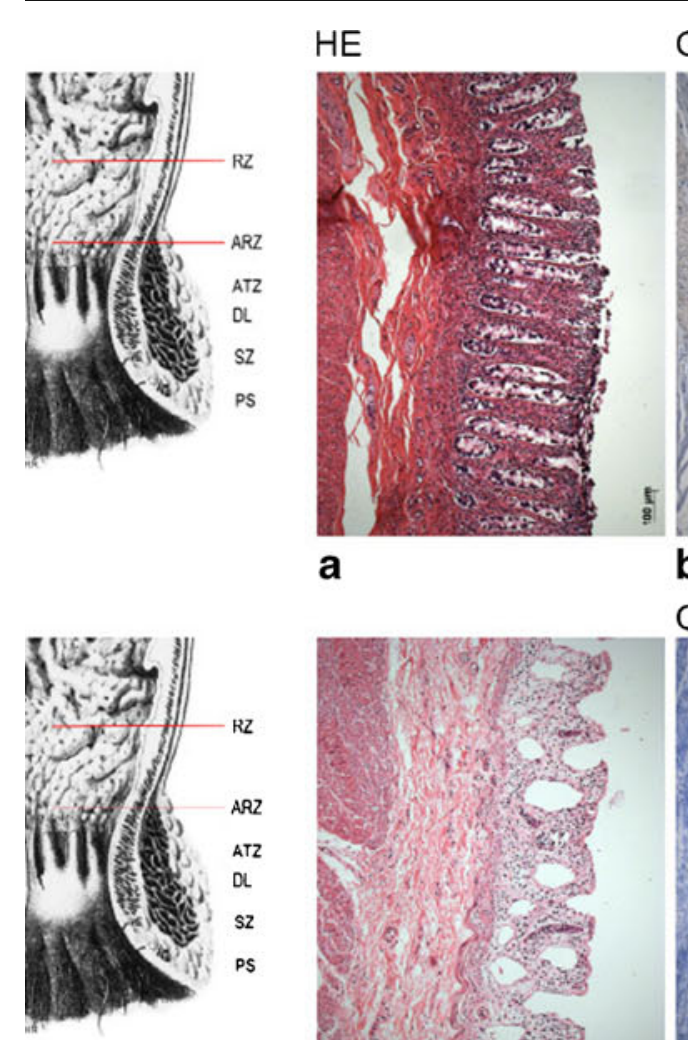

a

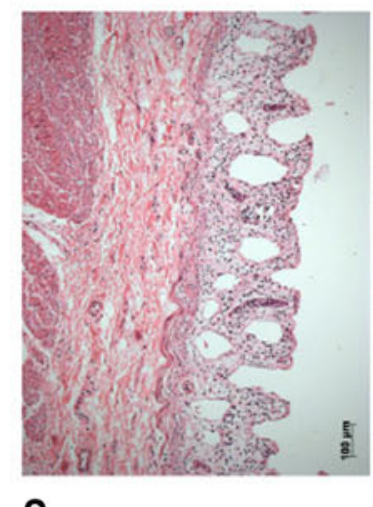

$$
\text { e }
$$

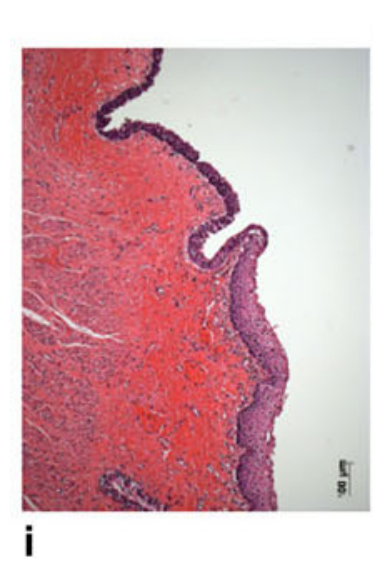

f

CK7

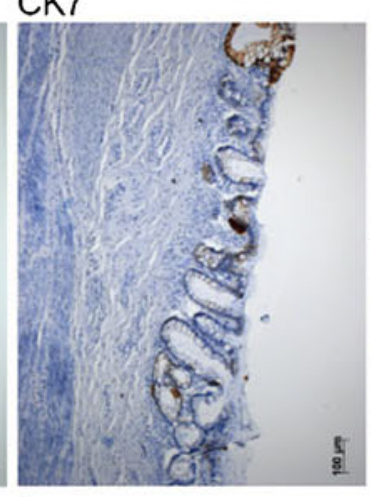

CK7
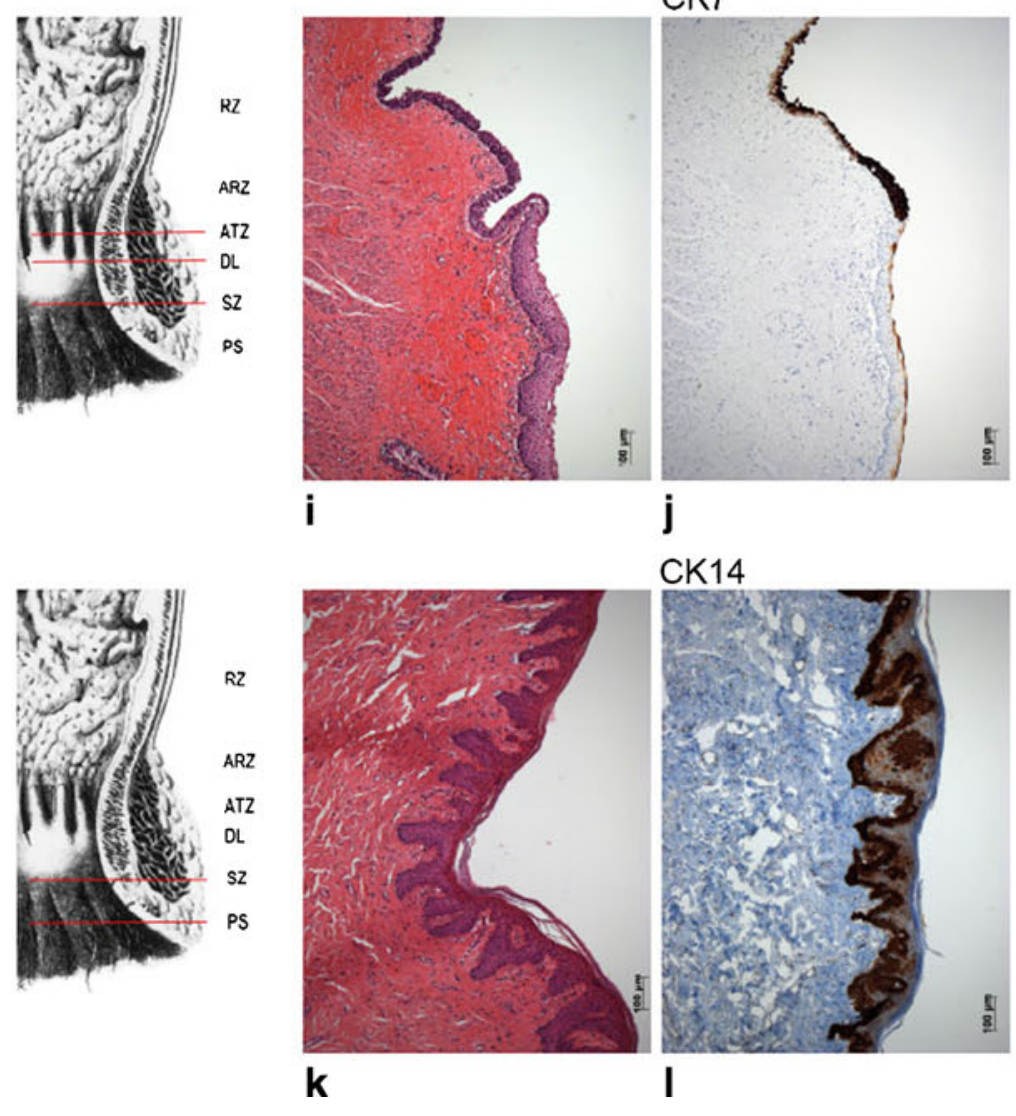
Fig. 6 a-l: Series of sagittal sections of a 65-year-old man. a-d RZ, e-h ARZ, $\mathbf{i}-\mathbf{j}$ ATZ, $\mathbf{k}-\mathbf{l}$ SZ and PS

developmental programme seems to take effect thataccording to the findings of Gao et al. [32] - is regulated by $\mathrm{Cdx} 2$ in mice. These authors showed that the intestinal growth is severely affected in $\mathrm{Cdx} 2$ mutant mice, and that $\mathrm{Cdx} 2$ deficiency prevents colon and rectal formation and leads to complete obstruction. New results in mice point to the fact that $\mathrm{Cdx} 2$ opposes tumour cell spreading [33]. We have shown that CDX2-positive cells are found in the bottom of healthy human rectal crypts in prenatal stages and in the adult where according to literature they play an important role in the regulation and differentiation of regular intestinal cells $[18,34]$. According to our results, we suppose that the typical rectal adenocarcinoma arises from the RZ and may arise from the ARZ, if scattered and regular crypts are found there. However, it does not arise from the ATZ. Our study reveals the possibility to demarcate and assign epithelial zones within the anorectum by means of immunohistochemistry. We could not determine any difference in $\mathrm{CK}$ expression among the different tumour stages. However, studies including a larger number of patients and different stages of rectal cancer as well as carcinomas of the anal squamous epithelium are required to analyze a possible changing in the $\mathrm{CK}$ and/or CDX2 expression pattern.

\section{Conclusion}

It is of importance to see that we have a distinct relation between the cell development in human embryos/fetuses and that of tumour cells, and that the knowledge of the regional features and their differentiation stages matters more than their affiliation with the so-called germ layers,
Fig. 7 a-d Series of transversal sections through an ultralow rectal adenocarcinoma (pT3, N0, M0, GIII). e-h Series of transverse sections through an anal squamous tumour (pT2, N0, M0, GII)

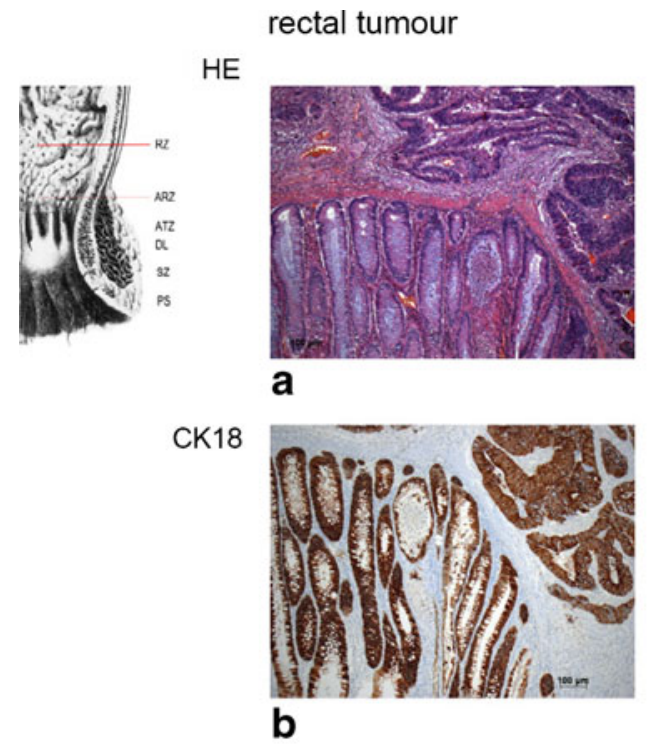

CK7

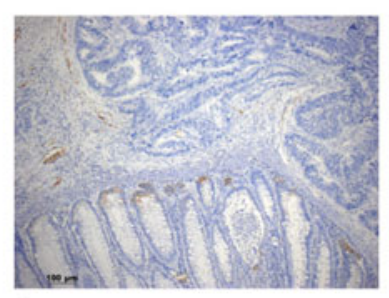

C

CDX2

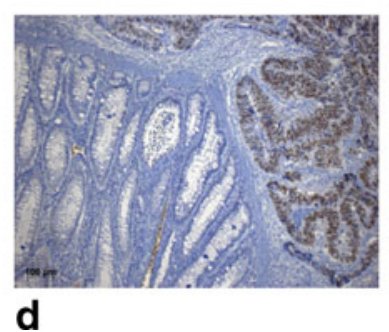

anal tumour

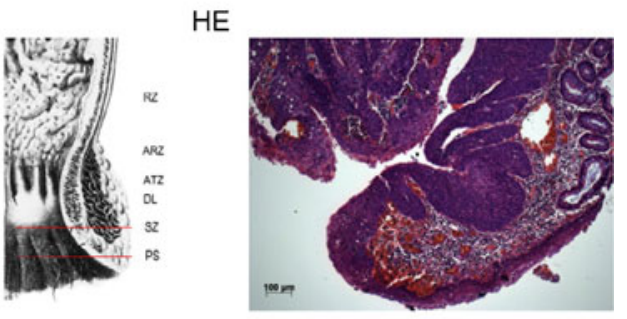

e

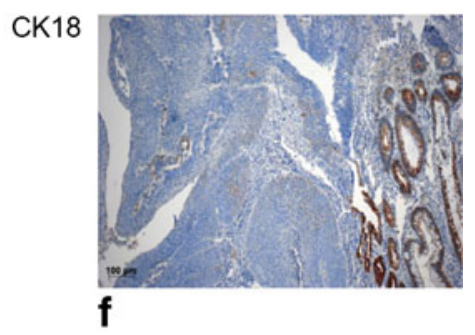

CK7

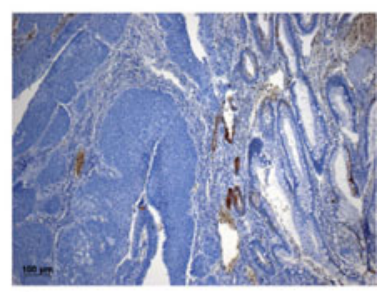

g

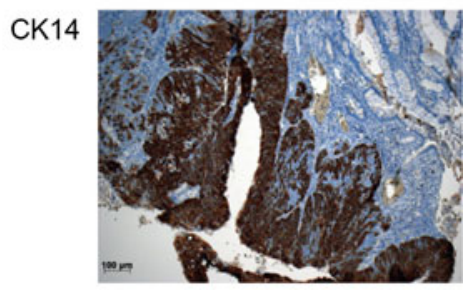

h 
which is in accordance with the statement that "the use of the term germ layers is critical and vague" as was already postulated in Gray's Anatomy [8].

Acknowledgements We are indebted to Elisabeth Richter for excellent technical assistance and to Romed Hörmann for excellent photographic assistance. We thank Claudia Siemon for helpful corrections of the English language.

Declaration of interest The authors declare that there is no conflict of interest that could be perceived as prejudicing the impartiality of the research reported.

Open Access This article is distributed under the terms of the Creative Commons Attribution Noncommercial License which permits any noncommercial use, distribution, and reproduction in any medium, provided the original author(s) and source are credited.

\section{References}

1. Böcker W, Denk H, Heitz PU (1996) Pathologie. Urban \& Fischer

2. Grabenbauer GG, Kessler H, Matzel KE, Sauer R, Hohenberger W, Schneider ICH (2005) Tumor site predicts outcome after radiochemotherapy in squamous-cell carcinoma of anal region: long-term results of 101 patients. Dis Colon Rectum 48:1742-1751

3. Hermanek P, Merkel S (2007) Pathology and classification of anal carcinomas. Der Onkologe 13:982-992

4. Fenger C (1997) Anal canal. In: Sternberg SS (ed) Histology for Pathologists. Lippincott-Raven Publishers

5. Johnson FP (1914) The development of the rectum in the human embryo. Am J Anat 16:1-57

6. O’Rahilly R, Müller F (1999) Embryologie und Teratologie des Menschen. $1^{\text {st }}$ German edition. Verlag Hans Huber

7. Sadler T (2004) Langman's medical embryology. Lippincott Williams and Wilkins Philadelphia Baltimore New York London Buenos Aires Hong Kong Sydney Tokyo pp. 313-317

8. Standring S (2005) editor in chief. Gray`s Anatomy. 39th ed. Elsevier Churchill Livingstone

9. Stelzner F (1998) Chirurgie an viszeralen Abschlusssystemen. Georg Thieme Stuttgart New York

10. Fritsch H, Aigner F, Ludwikowski B, Reinstadler-Zankl S, Illig R, Urbas D, Schwarzer C, Longato S (2007) Epithelial and muscular regionalization of the human developing anorectum. Anat Rec 290:1449-1458

11. Bernal SD, Stahel RA (1985) Cytoskeleton-associated proteins: their role as cellular integrators in the neoplastic process. Crit Rev Oncol Hematol 3:191-204

12. Moll R, Franke WW, Schiller DL, Geiger B, Krepler R (1982) The catalogue of human cytokeratins: patterns of expression in normal epithelia, tumors and cultured cells. Cell 31:11-24

13. Ramaekers F, Puts J, Kant A, Moesker O, Jap P, Vooijs P (1982) Differential diagnosis of human carcinomas, sarcomas and their metastases using antibodies to intermediate-sized filaments. Eur J Cancer Clin Oncol 18:1251-1257

14. Tseng SC, Jarvinen MJ, Nelson WG, Huang JW, WoodcockMitchell J, Sun TT (1982) Correlation of specific keratins with different types of epithelial differentiation: monoclonal antibody studies. Cell 30:361-372
15. Sun TT, Eichner R, Nelson WG et al (1983) Keratin classes: molecular markers for different types of epithelial differentiation. J Invest Dermatol 81:109-115

16. Moll R (1993) Cytokeratins as markers of differentiation. Expression profiles in epithelia and epithelial tumors. Veroeff Pathol 142:1-197

17. Erol A, Baskin LS, Li YW, Liu WH (2000) Anatomical studies of the urethral plate: why preservation of the urethral plate is important in hypospadias repair. BJU Int 85:728-734

18. Ee HC, Erler T, Bhathal PS, Young GP, James RJ (1995) Cdx-2 homeodomain protein expression in human and rat colorectal adenoma and carcinoma. AJP 147:586-592

19. Kaimaktchiev V, Terraccioano L, Tornillo L, Spichtin H, Stoios D, Bundi M, Korcheva V, Mirlacher M, Loda M, Sauter G, Corless CL (2004) The homeobox intestinal differentiation factor CDX2 is selectively expressed in gastrointestinal adenocarcinomas. Mod Pathol 17:1392-1399

20. Werling RW, Yaziji H, Bacchi CE, Gown AM (2003) CDX2, a highly sensitive and specific marker of adenocarcinomas of intestinal origin; an immunohistochemical survey of 476 primary and metastatic carcinomas. Am J Surg Pathol 27:303-310

21. Barbareschi M, Murer B, Colby TV, Chilosi M, Macri E, Loda M, Doglioni C (2003) CDX-2 homeobox gene expression is a reliable marker of colorectal adenocarcinoma metastases to the lungs. Am J Surg Pathol 27:141-149

22. de Vries PA, Friedland GW (1974) The staged sequential development of the anus and rectum in human embryos and foetuses. J Pediatr Surg 9:755-769

23. van der Putte SC (2005) The development of the perineum in the human. A comprehensive histological study with a special reference to the role of the stromal components. Adv Anat Embryol Cell Biol 177:1-131

24. van der Putte SC (2009) The development of the human anorectum. Anat Rec 292:951-954

25. Patten BM (1953) Human Embryology, 2nd edn. Mc Graw-Hill Book Company

26. Kromer P, Korzeniowska-Kromer E (2003) Anal canal development in the embryonic and early foetal period. Folia Morphol (Warsz) 62:285-287

27. Hollinshead WH (1956) Anatomy for surgeons: the thorax, abdomen and pelvis. Hoeber-Harper

28. Yamaguchi K, Kiyokawa J, Akita K (2008) Developmental process and ectodermal contribution to the anal canal in mice. Ann Anat 190:119-128

29. Saad RS, Silverman JF, Khalifa MA, Rowsell C (2009) CDX2, cytokeratins 7 and 20 immunoreactivity in rectal adenocarcinoma. Appl Immunhistochem Mol Morphol 17:196-201

30. Hyder SA and Cunningham C (2010) Malignant tumours of the anal canal and margin. In: Givel JC, Mortensen NJ, Roche B (eds.) Anorectal and colonic diseases. A practical guide to their management. $3^{\text {rd }}$ edition. Springer-Verlag Berlin Heidelberg

31. Goel A, Gupta I, Joshi K (1997) Immunohistochemical analysis of human embryos and fetuses: an insight into the mechanism of subversion of antigenic differentiation in neoplasia. Arch Pathol Lab Med 121:719-723

32. Gao N, White P, Kaestner KH (2009) Establishment of intestinal identity and epithelial-mesenchymal signalling by Cdx2. Dev Cell 16:588-599

33. Gross I, Duluc I, Benameur T, Calon A, Martin E, Brabletz T, Kedinger M, Domon-Dell C, Freund JN (2008) The intestinespecific homeobox gene $\mathrm{Cdx} 2$ decreases mobility and antagonizes dissemination of colon cancer cells. Oncogene 27:107-115

34. Bakaris S, Cetinkaya A, Ezberci F, Ekerbicer H (2008) Expression of homedomain protein $\mathrm{CDX} 2$ in colorectal adenoma and adenocarcinoma. Histol Histopathol 23:1043-1047 